\title{
Psychiatric Morbidity among Medical Out-Patients in Kuwait: Evaluation of a Somatic Inventory to Identify the Psychiatric from the Non-Psychiatric Patients
}

\author{
M.A. Zahida M.A.A. Motaal ${ }^{b}$ M.A. Razik ${ }^{b}$ \\ aDepartment of Psychiatry, Faculty of Medicine, Health Sciences Center, Kuwait University, and \\ bHospital for Psychological Medicine, Kuwait
}

\section{Key Words}

Psychiatric morbidity - Bradford Somatic Inventory (BSI) · Somatic symptoms

\begin{abstract}
Objectives: The aim of this study was to evaluate the Bradford Somatic Inventory (BSI), a 21-item questionnaire, to identify psychiatric patients attending a general hospital medical clinic in Kuwait. Methods: The sample consisted of 100 new general hospital medical outpatient clinic attendees. All patients were administered the BSI, and their psychiatric status was established by administration of the Present State Examination. All the patients who met the criteria of the International Classification of Diseases, 10th revision (ICD-10), were grouped as 'psychiatric' while the remaining subjects were regarded as 'non-psychiatric' patients. The frequency of endorsement and the total BSI scores were computed to determine sensitivity, specificity and positive predictive values of the BSI. Results: Fifty-one of the psychiatric patients met the ICD-10 diagnostic criteria. The psychiatric group reported a significantly higher number of BSI symptoms and had a significantly higher mean BSI total score than the non-psychiatric patients. Using a cut-off
\end{abstract}

score of $13 / 14$, the BSI-21 yielded a sensitivity rate of $72.5 \%$ and a specificity rate of $73.47 \%$. Evaluation of a shorter version, the BSI-15, comprising the most frequently endorsed items by the psychiatric patients, revealed similar results. Conclusions: The findings suggest that the $\mathrm{BSI}-21$ is a potentially useful screening instrument to identify psychiatric patients attending general medical out-patient clinics. The shorter version, BSI-15, is easier to administer and equally effective in discriminating the psychiatric from the non-psychiatric group of medical patients.

Copyright @ 2001 S. Karger AG, Basel

\section{Introduction}

A considerable proportion of patients seen in the general medical out-patient clinics are known to suffer from psychiatric rather than or in addition to a physical disorder $[1,2]$. Despite wide variation in the diagnostic criteria used and the population of the patients studied, there seems to be a general consensus that as many as $40-50 \%$ of the general medical out-patients may be suffering from psychiatric rather than or in addition to their physical illnesses [3, 4]. In the first part of this study, the presence of

\begin{tabular}{ll}
\hline KARGER & ( 2001 S. Karger AG, Basel \\
$1011-7571 / 01 / 0101-0023 \$ 17.50 / 0$ \\
$\begin{array}{l}\text { Fax }+41613061234 \\
\begin{array}{l}\text { E-Mail karger@karger.ch } \\
\text { www.karger.com }\end{array}\end{array}$ & $\begin{array}{l}\text { Accessible online at: } \\
\text { www.karger.com/journals/mpp }\end{array}$
\end{tabular}

Dr. M.A. Zahid, Associated Professor

Department of Psychiatry, Faculty of Medicine

Health Sciences Center, Kuwait University, PO Box 24923

13110 Safat (Kuwait)

Tel. +965 5312300 ext 6321, Fax +965 5338904 
psychiatric morbidity among general hospital medical out-patient clinic attendees was described [5]. Based on the administration of the Present State Examination (PSE), 51 out of 100 medical out-patients met the International Classification of Diseases, 10th revision (ICD-10), diagnostic criteria for psychiatric disorders [6, 7]. It was found that generalised anxiety disorder, mild depressive disorder and mixed anxiety and depressive disorder constituted more than two thirds of the psychiatric disorders while irritable bowel syndrome, peptic ulcer, hypertension and gastritis were the commonest physical diagnoses assigned to these patients by their respective physicians. In this part of the study, the usefulness of the Bradford Somatic Inventory (BSI) [8] in distinguishing the 'psychiatric' from the 'non-psychiatric' group of patients is discussed.

Various instruments have been devised to detect psychiatric morbidity amongst medically ill patients. It has been suggested that some of the instruments for measurement used in the psychiatric population of patients, especially those suffering from mood disorders, may not be valid in patients with co-existing medical conditions [9, 10]. This is because the somatic symptoms like poor sleep, poor appetite, lethargy and poor concentration on which these instruments rely do not necessarily indicate the presence of a psychiatric disorder and, in fact, can be directly attributed to the underlying physical pathology [11]. The instruments designed to measure psychiatric morbidity among the medical clinic attendees, including the Hospital Anxiety and Depression Scale [12] and the modified DSM-IV (Diagnostic Statistical Manual, 4th edition) criteria as proposed by Endicott [13] therefore exclude somatic symptoms to minimise the risk of falsepositive results in this group of patients. There is, however, one problem with this assessment procedure. Mooddisordered patients who somatise their symptoms are likely to be missed if the measuring instrument relies heavily on psychological rather than or in addition to somatic symptoms. It is becoming increasingly clear that somatic symptoms are a very common mode of presentation of psychiatric illnesses [14]. The somatic language, being the least vulnerable to changes in the meaning of words, has been regarded by some as the most vivid way of communicating emotions [15].

A number of inventories including the Vietnamese Depression Scale [16], Nigerian Scale of Somatic Symptoms [17], Chinese Health Questionnaire [18] and the BSI, [8] have been developed to measure the psychiatric morbidity among psychologically disturbed patients presenting with somatic symptoms. Consisting mainly of somatic symptoms, most of such inventories have been designed to be appropriate to particular ethnic groups. The BSI was designed to meet the need for a multi-ethnic somatic inventory. It was constructed through a systematic search into the case-notes of patients presenting to medical clinics without evidence of physical disease. Its initial 46-item version covered more than $90 \%$ of the symptoms reported by the patients across five different centres, including North and South India, Nepal, Pakistan and Britain [8]. Later validation studies in Pakistan and Britain revealed that shorter versions, BSI-21 and BSI-14, were equally effective and easy to administer [19].

The predominance of somatic over behavioural and emotional presentations of psychiatric disorders in the Arab communities has been well documented [20]. We, therefore, decided to (a) evaluate the usefulness of the BSI-21 in detecting psychiatric morbidity among general medical out-patients in Kuwait, and (b) determine the relative usefulness of its items in distinguishing such patients.

\section{Methods}

\section{Sampling}

The nursing staff, stationed in the out-patient clinic and concerned with the registration of new patients, was requested to refer all new consenting patients for psychiatric assessment. The patients were assessed on two different weekdays, spread evenly throughout the 18 -week study period. Circumstances surrounding the recruitment of patients did not permit psychiatric evaluation of a number of possibly consenting patients and randomisation of the patients was not possible. This was because the general medical out-patient clinics were overcrowded, drawing 50-100 patients daily while a maximum of only 2 patients could be seen at one given time. One hundred patients were recruited, about $5-7 \%$ of all the new out-patient clinic attendees.

\section{Procedures}

All the patients were administered a semi-structured psychiatric interview and the BSI. Both of these examinations were conducted before the physicians' consultation. The psychiatric interview administered by two of the co-authors, M.A.R. and M.M.A.M., who are trained psychiatrists, was based on obligatory questions from the PSE: The questions related to 'neurotic, stress-related and somatoform disorders', the commonest psychiatric conditions described in medical out-patient settings [2-4].

Translation of the BSI-21. The BSI-21 was translated into Arabic by a non-practising physician. It was then backtranslated into English by two trained psychiatrists. All three translators discussed the discrepancies in the translated versions. This led to recording of some and rephrasing of other BSI items to achieve the maximum possible conceptual equivalence [21] between the English and Arabic versions. 
Administration of the BSI. The BSI was originally designed and validated as a self-reporting questionnaire. In order to include the illiterate and those patients, especially expatriates, who are unable to complete the questionnaire either in Arabic or English, the questionnaire was rater administered. The raters were instructed to confine themselves to asking the question verbatim and not offer explanations of any sort. None of the questions went unanswered though some of the patients had to think hard whether they had experienced the symptom for more or less than 15 days during the past month. Each patient was administered the BSI- 21 by a psychologist. The psychologist was blind to the outcome of the psychiatric interview.

Each BSI item presents a three-choice format: (1) absent; (2) present on less than 15 days during the past month; (3) present on more than 15 days during the past month. These responses are scored 0,1 and 2 , respectively. The total score (range $0-42$ ) was derived by adding the score of each of the 21 items. An alternate scoring system, counting only those BSI symptoms which had been present for more than 15 days during the past month, used and successfully validated in the previous studies [19], was also computed.

\section{Statistical Analyses}

Analysis of variance (ANOVA) was used to measure the total number of BSI symptoms and the total BSI scores of both groups of patients.

The frequency of endorsement of each of the 21 BSI items by both groups, the psychiatric and the non-psychiatric patients, was computed. $\chi^{2}$ tests were conducted to determine the significance of the difference in each item endorsement and the total BSI scores between the two groups.

The results of a validation study of a psychiatric screening questionnaire are usually presented in the form of single values for the sensitivity and the specificity (the proportion of 'true cases' and of 'true non-cases' correctly identified by the instrument). Lowering the cut-off score increases sensitivity at the expense of specificity while raising the cut-off score increases specificity at the expense of sensitivity. In order to achieve the optimum balance between sensitivity and specificity, different cut-off scores were computed to assign group status (psychiatric versus non-psychiatric), identified on the basis of their BSI scores, to the patients.

For practising clinicians to confidently use the BSI to screen for psychiatric morbidity, the most important criterion would be the 'positive predictive value'. This is the likelihood that someone with a high score will turn out to be a 'case' and is highly dependent on the prevalence of the disorders in the population surveyed [22]. Assuming a prevalence of psychiatric disorders of 30\% [22], the positive predictive value of the BSI to discriminate between psychiatric and non-psychiatric cases was computed.

The data were analysed on SPSS; version 8.0.

\section{Results}

The sample studies consisted of 100 new medical outpatients. Fifty-one of these patients met diagnostic criteria for psychiatric disorders while the remaining 49 cases were regarded as non-psychiatric cases.
Table 1. Mean BSI symptoms and total score

\begin{tabular}{|c|c|c|}
\hline & $\begin{array}{l}\text { Psychiatric } \\
\text { group }\end{array}$ & $\begin{array}{l}\text { Non-psychiatric } \\
\text { group }\end{array}$ \\
\hline $\begin{array}{l}\text { Number of symptoms endorsed } \\
\text { BSI Score }\end{array}$ & $11.69(4.36)$ & $6.45(4.52)$ \\
\hline BSI-21 & $17.67(6.72)$ & 8.59 (6.49) \\
\hline BSI-15 & $13.65(5.13)$ & $6.49(5.06)$ \\
\hline
\end{tabular}

Figures in parentheses indicate SD.

\section{BSI Symptom Endorsement: Psychiatric versus \\ Non-Psychiatric Patients}

Analyses of variance indicated that the psychiatric group reported a significantly higher number of symptoms on the BSI $[\mathrm{F}(1,98)=34.75, \mathrm{p}<0.001]$ and had a significantly higher mean BSI total score $[\mathrm{F}(1,98)=47.13$, $\mathrm{p}<0.001]$ than the non-psychiatric group, Table 1 presents the mean number of symptoms endorsed and the mean BSI total score by group.

\section{Selection of Shorter Version: BSI-15}

Table 2 shows the frequency of endorsement of BSI items by the psychiatric versus non-psychiatric groups. None of the 21 items were endorsed by more than $49 \%$ of the non-psychiatric patients. In contrast, the majority of the psychiatric patients endorsed several of the 21 items. Fifteen items were endorsed significantly more frequently by the psychiatric group of patients. These 15 items were taken as constituting the shortened version of the inventory, the 'BSI-15'.

When the other scoring system (counting only those BSI symptoms which were present for more than 15 days during the past month) was used, 13 items were endorsed significantly more frequently by the psychiatric than the non-psychiatric group. These included all but 2 (No. 15: heavy head, No. 19: heart weak or sinking) of the BSI-15 items.

\section{Evaluation of the BSI-21}

Calculation of sensitivity and specificity using different cut-off scores revealed an optimum cut-off score of $13 / 14$ which yielded a sensitivity rate of $72.5 \%$ (37 out of 51 psychiatric cases) and a specificity rate of $73.47 \%$ (36 out of 49 non-psychiatric cases). Table 3 presents the number of 'true-positive' and 'true-negative' results using this cut-off score. Using the 13/14 cut-off score, the BSI21 yielded a positive predictive value of 53-60\%. 
Table 2. BSI: percentage endorsement by psychiatric and non-psychiatric patients

\begin{tabular}{|c|c|c|c|c|}
\hline \multirow[t]{2}{*}{ BSI items } & \multicolumn{2}{|c|}{$\begin{array}{l}\text { Psychiatric group } \\
(\mathrm{n}=51)\end{array}$} & \multicolumn{2}{|c|}{$\begin{array}{l}\text { Non-psychiatric group } \\
(\mathrm{n}=49)\end{array}$} \\
\hline & $\mathrm{n}$ & $\%$ & $\mathrm{n}$ & $\%$ \\
\hline 1 Severe headaches** & 38 & 74.5 & 36.7 & 18 \\
\hline 2 Stomach fluttering* & 62.8 & 32 & 32.7 & 16 \\
\hline 3 Neck pain or tension* & 29 & 56.8 & 34.7 & 17 \\
\hline 4 Head constriction* & 28 & 54.9 & 20.4 & 10 \\
\hline 5 Chest pain & 29 & 56.9 & 34.6 & 17 \\
\hline 6 Dry mouth** & 35 & 68.6 & 40.9 & 20 \\
\hline 7 Weakness or anergy** & 45 & 88.3 & 49.0 & 24 \\
\hline 8 Sweating a lot* & 19 & 37.2 & 16.3 & 8 \\
\hline 9 Chest pressure** & 62.7 & 32 & 28.5 & 14 \\
\hline 10 Choking sensation & 45.1 & 23 & 24.5 & 12 \\
\hline 11 Total body aches and pains* & 58.9 & 30 & 26.5 & 13 \\
\hline 12 Palpitations & 68.7 & 34 & 42.8 & 21 \\
\hline 13 Trembling or shaking & 21 & 41.2 & 24.5 & 12 \\
\hline 14 Urine frequency* & 28 & 54.9 & 30.6 & 15 \\
\hline 15 Head heavy* & 49.0 & 25 & 22.5 & 11 \\
\hline 16 Tired all the time* & 52.9 & 27 & 28.5 & 14 \\
\hline 17 Head about to burst* & 41.2 & 21 & 14.3 & 7 \\
\hline 18 Constipation & 43.1 & 22 & 38.8 & 19 \\
\hline 19 Heart weak or sinking* & 31.4 & 16 & 8.2 & 4 \\
\hline 20 Excessive wind or gas* & 82.4 & 42 & 59.2 & 29 \\
\hline 21 Hands or feet cold & 39.2 & 20 & 30.6 & 15 \\
\hline
\end{tabular}

$* \mathrm{p}<0.05,{ }^{* *} \mathrm{p}<0.01$.

Items $1-4,6-9,11,14-17,19$ and 20 were included in the BSI-15.

Table 3. Predicted group membership using the BSI total score

\begin{tabular}{|c|c|c|c|c|}
\hline \multirow{3}{*}{$\begin{array}{l}\text { Membership } \\
\text { actual group }\end{array}$} & \multicolumn{4}{|l|}{ Predicted group } \\
\hline & \multicolumn{2}{|l|}{ psychiatric } & \multicolumn{2}{|l|}{ non-psychiatric } \\
\hline & BSI-21 & BSI-15 & BSI-21 & BSI-15 \\
\hline Psychiatric $(\mathrm{n}=51)$ & $\begin{array}{l}72.5 \%(\mathrm{n}=37) \\
\text { (true-positive) }\end{array}$ & $\begin{array}{l}70.6 \%(\mathrm{n}=36) \\
\text { (true-positive) }\end{array}$ & $\begin{array}{l}27.5 \%(n=14) \\
\text { (false-negative) }\end{array}$ & $\begin{array}{l}29.4 \%(\mathrm{n}=15) \\
\text { (false-negative) }\end{array}$ \\
\hline Non-psychiatric $(\mathrm{n}=49)$ & $\begin{array}{l}26.5(\mathrm{n}=13) \\
\text { (false-positive) }\end{array}$ & $\begin{array}{l}22.4 \%(\mathrm{n}=11) \\
\text { (false-positive) }\end{array}$ & $\begin{array}{l}73.5 \%(\mathrm{n}=36) \\
\text { (true-negative) }\end{array}$ & $\begin{array}{l}77.6 \%(\mathrm{n}=38) \\
\text { (true-negative) }\end{array}$ \\
\hline
\end{tabular}

\section{Evaluation of the Shorter Version BSI-15}

For ease of administration, the feasibility of using a shortened version of the BSI was assessed using 15 items of the BSI. The selection of the items, as described earlier, was based on the frequency of their endorsement by the patients. Subjects' scores on the BSI-15 were computed in the same way as the 21-item BSI scores. The total BSI-15 score was obtained by adding the score $(0,1$ or 2$)$ on each of the 15 items. Also listed in table 1 are the means of the BSI-15 scores of the psychiatric and the non-psychiatric groups. The psychiatric group obtained a significantly higher mean score $[F(1,98)=49.24, p<0.001]$ than the non-psychiatric group. Calculation of sensitivity and specificity of BSI-15 using different cut-off scores revealed an optimum cut-off point of $9 / 10$ which yielded a sensitivity rate of $70.58 \%$ (36 out of 51 psychiatric cases) and a specificity rate of 77.55 ( 38 out of 49 non-psychiatric cases) as shown in table 3 . Using the same 13/14 cutoff score, the BSI-15 yielded a similar range for positive predictive value. 


\section{Discussion}

The ability of the BSI to distinguish the psychiatric from the non-psychiatric group of our patients hinged on two factors: the efficacy of the BSI to accurately depict the somatic symptoms likely to be psychiatric in origin and the accuracy of the psychiatric diagnoses. The psychiatric group endorsed a significantly higher number of symptoms on the BSI and had a significantly higher mean BSI total score than the non-psychiatric group. Similarly, the calculation of sensitivity and specificity rates revealed the BSI to be a potentially useful instrument in identifying somatic symptoms reported by the psychiatric (as opposed to non-psychiatric) group. The patients' group status, psychiatric or non-psychiatric, was established on the basis of a standardised psychiatric interview, and diagnoses were assigned according to the ICD-10. Therefore, it can be safely deduced that psychiatric diagnoses were assigned with a fair amount of accuracy.

The basis on which the BSI might be able to identify psychiatric patients is threefold: (a) the accurate characterisation (psychiatric vs. non-psychiatric) of somatic symptoms; (b) the wider spectrum of symptoms reported by the psychiatric patients, and (c) the time course of particular symptoms. The items of the BSI have been derived from a catalogue of somatic symptoms, most commonly reported by the psychiatric patients presenting predominantly, if not exclusively, with somatic symptoms [8]. It is characteristic of many patients presenting with somatic symptoms not of organic nature that their complaints are not confined to one region or system of the body. Both the BSI-2 1 and BSI-15 include symptoms from the head, abdomen, chest and heart, and skeletal muscles. Since the somatic symptoms reported by psychiatric patients are likely to be more chronic than symptoms of many physical illnesses [23], a duration-weighted scoring system has been adopted to score the BSI items. The symptoms reported for more than 15 days are scored as 2 while those reported for less than 15 days are scored as 1 .

In a previous comparative study, the screening power of the BSI was compared with the Hospital Anxiety and Depression (HAD) Scale, an instrument widely used to measure psychiatric morbidity among the medical clinic attendees [12]. The sensitivity and specificity rates and the positive predictive value of the BSI corresponded closely to those of the HAD [19]. Similarly, in a large validation study comprising 600 medical out-patients, the BSI was successfully used to discriminate 'non-organic' (psychiatric) from the 'organic' (non-psychiatric) group of somatic symptoms [19, 23].

Psychiatric Morbidity among Medical

Patients
The clinical presentation of emotional distress and social problems in somatic terms (somatisation) has been shown to be common in all culture groups and societies studied [14, 24]. El-Islam [20], in his review of psychiatry in the Arab culture, has found somatic presentation of behavioural and emotional disturbances to be fairly common among the Arab communities. More than half of our patients were found to have psychiatric disorders. Numerous studies have reported that psychiatric disorders not only lead to over-utilisation of medical services but also adversely affect the prognosis in the medically ill patients $[25,26]$.

The findings suggest that both BSI-21 and BSI- 15 can usefully be employed to identify the psychiatric patients presenting in the medical clinics. The BSI-15 has the advantage of being shorter and, therefore, easier to administer. It takes only 5-7 minutes to administer and it is available in three different languages: Urdu, English and Arabic. Since it relies on the physical symptoms most commonly reported by the psychologically disturbed patients, primary health care centres and a variety of medical settings including cardiology, nephrology, oncology, gastro-enterology and dermatology clinics are other potential sites where the BSI can be employed to distinguish psychiatric from the non-psychiatric group of patients attending these clinics. The positive predictive value of $53-60 \%$ for the BSI- 15 needs to be compared with other screening instruments. Goldberg [22] has reviewed data from all the principal tests used to screen psychiatric illness among medical patients, standardising them to $30 \%$ prevalence. The resulting positive predictive values ranged from 52 to $82 \%$, which puts the BSI in the same range.

Lastly, there were some methodological limitations of the study: (a) the size of the sample was small; (b) the circumstances of the study made it impossible to recruit all new consecutive patients or a truly randomised sample because the clinics were crowded and only 2 patients could be examined at a time, and (c) the sample consisted of about $5-7 \%$ of all the new medical out-patient clinic attendees during the study period. Therefore, it is probable that there was some sampling bias. Secondly, the BSI was originally designed and validated as a self-administered questionnaire but in our study it was administered by the clinical psychologists. This was done, as suggested in an earlier validation study [19], to include the illiterate patients. A previous study [27] suggested a drop of 30\% in the BSI score when administered by a doctor, but this study was carried out on medical students and may not necessarily imply a similar drop in the patients likely to have experienced the symptoms assessed. 


\section{Conclusions}

The results of this preliminary validation study suggest that both the BSI-21 and BSI-15 are useful screening instruments to distinguish the psychiatric from the nonpsychiatric group of general hospital medical out-patients. The shorter version (BSI-15) is easier to administer and is equally effective. Primary health care centres and medical settings like cardiology, nephrology, gastro-enterology and dermatology clinics are other potential sites where the BSI can be used to distinguish the psychiatric from the non-psychiatric group of patients attending these clinics.

\section{Acknowledgement}

This study was funded by Kuwait University grant No. MDQ 283.

\section{References}

1 Goldberg D, Privett M, Ustan B, Simon G, Linden M: The effects of detection and treatment on the outcome of major depression in primary care: A naturalistic study in 15 cities. Br J Gen Pract 1998;48:1840-1844.

2 Jacobsson L: Psychiatric morbidity and psychological background in an outpatient population of a general hospital in western Ethiopia. Acta Psychiatr Scand 1985;71:417-426.

3 Ruiz A, Silva H: Prevalence of psychiatric disorders in an outpatient service of general medicine. Rev Med Chil 1990;118:339-345.

4 Barsky AJ, Cleary PD, Coeytaux RR, Ruskin $\mathrm{JN}$ : Psychiatric disorders in medical outpatients complaining of palpitations. J Gen Intern Med 1994;9:306-313.

5 Zahid MA, Razik AA, Mohsin MAM: Psychiatric morbidity among medical outpatients in a general hospital. Med Principles Pract 1999;8:301-308.

6 Wing JK, Cooper JE, Sartorius N: The Measurement and Classification of Psychiatric Symptoms. London, Cambridge University Press, 1974.

7 International Classification of Diseases: Research Diagnostic Criteria, rev. 10. Geneva, WHO, 1992.

8 Mumford DB, Bavington JT, Bhatnagar KS, Hussain Y, Naraghi MS: The Bradford Somatic Inventory: A multi-ethnic inventory of somatic symptoms reported by anxious and depressed patients in Britain and the Indo-Pakistan subcontinent. Br J Psychiatry 1991;158. 379-386.

9 House A: Mood disorders in the physically ill: Problems of definition and measurement. J Psychosom Res 1989;32:345-353.
10 Williams P, Tarnopolsky A, Hand D: Case definition and case identification in psychiatric epidemiology: Review and assessment. Psychol Med 1980;10:101-104.

11 Ramasubbu R, Kennedy SH: Factors complicating the diagnosis of depression in cerebrovascular disease. II. Neurological deficits and various assessment methods. Can J Psychiatry 1994;39:601-607.

12 Zigmond AS, Snaith RP: The hospital anxiety and depression scale. Acta Psychiatr Scand 1983;67:361-370.

13 Endicott J: Measurement of depression in patients with cancer. Cancer 1984;53(suppl): 2243-2248.

14 Kirmayer LJ, Young A: Culture and somatization. Psychosom Med 1998;60:420-430.

15 Mumford DB: Emotional distress in the Hebrew Bible. Br J Psychiatry 1992;160:92-97.

16 Kinzie JD, Manson SM, Vinh DT, Tolan NT, Anh B, Pho TN: Development and validation of a Vietnamese language depression rating scale. Am J Psychiatry 1982;139:1276-1281.

17 Ebigdo PO: Development of a culture specific (Nigeria) screening scale of somatic complaints indicating psychiatric disturbance. Cut Med Psychiatry 1982;6:29-43.

18 Cheng T, Williams P: The design and development of a screening questionnaire (CHQ) for use in community studies of mental disorders in Taiwan. Psychol Med 1986;16:415-422.

19 Mumford DB, Tareen IAK, Bhatti MR, Bajwa MAZ, Ayub M, Pervaiz T: An investigation of 'functional' somatic symptoms among patients attending medical clinics in Pakistan. 11. Using somatic symptoms to identify patients with psychiatric disorders. J Psychosom Res 1991; 35:257-264.
20 El-Islam M: Arab cultural psychiatry. Transcult Psychiatr Res Rev 1982;19:5-24.

21 Brislin RW: Translation and content analysis of oral and written materials; in Triandis HE, Berry JW (eds): Handbook of Cross-Cultural Psychology. Methodology, Boston, Allyn \& Bacon, 1980, vol 2.

22 Goldberg D: Identifying psychiatric illness among general medical patients. Br Med J 1985;291:161-162.

23 Mumford DB, Tareen IAK, Bajwa MAZ, Bhatti MR, Pervaiz T, Ayub M: An investigation of functional somatic symptoms among patients attending medical clinics in Pakistan. I. Characteristics of 'non-organic' patients. J Psychosom Res 1991;35:45-255.

24 Isaac M, Janca A, Orley J: Somatization - A culture bound or universal syndrome? J Ment Health 1996;5:219-222.

25 Fink P: Mental illness and admission to general hospital. Acta Psychiatr Scand 1990;82:458462.

26 Vogt T, Pope C, Mulloly J, Hollis J: Mental health status as a predictor of morbidity and mortality: A 15-year follow-up of members of a health maintenance organisation. Am J Public Health 1994;84:227-231.

27 Mumford DB: Somatic sensations and psychological distress among students in Britain and Pakistan. Soc Psychiatry Psychiatr Epidemiol 1989;24:321-326. 